\title{
Polycomb chromobox Cbx2 enhances antiviral innate immunity by promoting Jmjd3-mediated demethylation of H3K27 at the Ifnb promoter
}

\author{
Donghao Sun, Xuetao Cao, Chunmei Wang ${ }^{\bowtie}$ \\ Department of Immunology and Center for Immunotherapy, Institute of Basic Medical Sciences, Peking Union Medical College, \\ Chinese Academy of Medical Sciences, Beijing 100005, China \\ $\triangle$ Correspondence: chunmeiwang@cams.cn (C. Wang) \\ Received June 26, 2018 Accepted September 28, 2018
}

\begin{abstract}
Polycomb chromobox (CBX) proteins regulate gene transcription by maintaining chromatin states, which guide a variety of biological processes. Now, epigenetic regulation of innate immune response is an emerging field. However, the role of CBX proteins in innate immunity remains unclear. We confirmed that the expression of $\mathrm{CBX}$ family proteins, especially $\mathrm{Cbx} 2$, was decreased in macrophages upon viral infection, and then we investigated the role of $\mathrm{Cbx} 2$ in the antiviral immune response. Silencing or knockdown of $\mathrm{Cbx} 2$ in macrophages inhibited virus-induced production of IFN$\beta$. Furthermore, heterozygous $\mathrm{Cbx} 2$ knockout were susceptible to VSV challenge. Mechanistically, Cbx2 binds to and recruits $\mathrm{Jmjd} 3$ to the Ifnb promoter, leading to demethylation of $\mathrm{H} 3 \mathrm{~K} 27 \mathrm{me} 3$ and increased transcription of IFN- $\beta$. Together, our study reveals a nontraditional function of a $\mathrm{Cbx}$ protein and adds new insight into the epigenetic regulation of antiviral innate immunity.
\end{abstract}

KEYWORDS Cbx2, Jmjd3, interferon beta, innate immunity

\section{INTRODUCTION}

The innate immune response is an important determinant in the fight against virus infection, deploying pattern-recognition receptors (PRRs), such as the RIG-I-like receptor (RLR), to

Electronic supplementary material The online version of this article (https://doi.org/10.1007/s13238-018-0581-0) contains supplementary material, which is available to authorized users. sense and respond to pathogens (Cao, 2016; Kawai and Akira, 2010). Upon binding to pathogen-associated molecular patterns (PAMPs), PRRs activate a series of signaling cascades, leading to the induction of type I interferons (IFNs) by nuclear factor kappa-light-chain-enhancer of activated $B$ cells (NF-KB), interferon regulatory factor (IRF) $3 / 7$ and/or activator protein-1 (AP-1) (Iwasaki and Pillai, 2014; Wu and Chen, 2014). Subsequently, IFNs induce the expression of interferon stimulated gene (ISGs) to restrict viral replication through the activation of the Janus kinase (JAK)-signal transducer and activator of transcription (STAT) pathway (McNab et al., 2015). Precise control of the innate immune response is critical for maintaining host immune homeostasis and ensuring proper viral clearance.

Epigenetic modifications such as DNA methylation, histone modifications, noncoding RNA regulation and chromatin remodeling have been shown to impact gene transcription. Epigenetic regulation in innate immunity has been studied extensively in recent years, yielding insights into the detailed molecular mechanisms that modulate the expression of inflammatory cytokines and type I interferons (Marcos-Villar et al., 2018; Yoshida et al., 2015; Zhang et al., 2015). For example, Tet2 recruits Hdac2 to specifically repress IL- 6 by histone deacetylation, which resolves inflammation (Zhang et al., 2015). Dnmt3a promotes activation of TBK1 by maintaining high expression of the histone deacetylase HDAC9, leading to the production of type I interferons and antiviral innate immunity (Li et al., 2016). The IncRNA Inc-Lsm3b binds to RIG-I monomers and restricts RIG-I protein's conformational shift, thereby terminating type I IFNs production (Jiang et al., 2018). However, the identification of other unknown epigenetic modifier and the underlying mechanisms for innate immunity remain to be further explored. 
The Polycomb group (PcG) proteins, which are best characterized by two protein complexes (Polycomb repressive complex 1 [PRC1] and Polycomb repressive complex 2 [PRC2] have been identified as critical regulators of gene expression that maintain repressive chromatin states (Bernstein et al., 2006; Simon and Kingston, 2013). Chromobox (CBX) family proteins are canonical components of PRC1 and are divided into two groups: HP1 $\alpha(\mathrm{Cb} \times 5)$, $\mathrm{HP} 1 \beta(\mathrm{Cbx} 1)$, and HP1Y(Cbx3); and $\mathrm{Cbx} 2, \mathrm{Cbx} 4, \mathrm{Cbx} 6$, $\mathrm{Cbx} 7$ and $\mathrm{Cbx} 8$. The latter group has an N-terminal chromodomain that serves as a recognition site and binds to H3K27me3, which helps to recruit and stabilize PRC1 to specific regions of the chromatin (Aranda et al., 2015; Di Croce and Helin, 2013). Accumulating evidence has demonstrated that $\mathrm{CBX}$ proteins play crucial roles in a variety of biological processes, such as the differentiation of stem cells, neurite development and tumor angiogenesis (Chen et al., 2017; Santanach et al., 2017; Sparmann and van Lohuizen, 2006). Recently, Cbx7 has been shown to repress activation-induced $T$ cell apoptosis, indicating a role in the adaptive immune response ( $\mathrm{Li}$ et al., 2014a, b). However, the role of CBX family proteins in innate immunity remains largely unknown.

In this report, we addressed the function of $\mathrm{Cbx} 2$ in antiviral innate immunity. Our results demonstrate that $\mathrm{Cbx} 2$ enhances the production of type I interferon by binding and recruiting Jmjd3 to the promoter of IFN- $\beta$, thus activating the antivirus immune response. Therefore, our work identifies Cbx2 as a positive mediator in antiviral immunity, which provides new mechanistic insight into epigenetic modifiers in the innate immune response.

\section{RESULTS}

Cbx2 promotes virus-induced production of type I interferon in macrophages

Gene profiling data indicate the decreased expression of CBX family proteins is observed after infection with virus in the brain (GEO: GSE44331) and in RAW246.7 macrophages (GEO: GSE81675), inspiring us to investigate the role of $C B X$ proteins in the innate immune response. We first examined the expression pattern of CBX proteins in macrophages upon virus infection. $A$ reduction in the expression of CBX, and especially Cbx2, was observed in VSV-infected macrophages (Fig. 1A), suggesting that CBX proteins may be involved in the antiviral immune response. Because the expression of $\mathrm{Cbx} 2$ is highest in macrophages compared with other immune cells, such as T cells and natural killer cells (Fig. 1B), we focused on investigating the role of $\mathrm{Cbx} 2$ in antiviral innate immunity. A decrease in Cbx2 mRNA and protein were observed upon infection of mouse peritoneal macrophages (Fig. 1C) and RAW264.7 macrophages
(Fig. 1D) with a variety of viruses in addition to VSV, and upon exposure to Poly (I:C), which suggested the decreased expression of $\mathrm{Cbx} 2$ might be involved in the antiviral innate immune response.

We used small interfering RNA (si-Cbx2) to silence Cbx2 expression in mouse peritoneal macrophages that were then infected with VSV (RNA virus) or HSV (DNA virus) (Fig. 2A). $A$ decrease in the mRNA level (Fig. 2B) and protein level (Fig. 2C) of IFN- $\beta$ was observed in Cbx2 knockdown macrophages infected with virus, as well as IFN- $\alpha$ and IL-6, but not TNF- $\alpha$ (Fig. S1A and S1B). Consistently, overexpression of $\mathrm{Cbx} 2$ (Fig. 2D) enhanced VSV or HSV-induced production of IFN- $\beta$ in RAW246.7 cells (Fig. 2E and 2F). These findings suggest that $\mathrm{Cbx} 2$ promotes antiviral innate immunity by enhancing the production of type I interferon.

\section{In vivo knockdown of $\mathrm{Cbx} 2$ increases susceptibility to VSV challenge}

Because Cbx2 gene knockout is developmentally lethal (Katoh-Fukui et al., 1998), we used Cbx2 heterozygous knockout mice $\left(\mathrm{Cbx} 2^{+/-}\right.$mice) to investigate the role of $\mathrm{Cbx} 2$ in the antiviral response in vivo. The in vivo reduction in Cbx2 expression levels in $\mathrm{Cbx}^{+/-}$mice was confirmed (Fig. 3A). When challenged with VSV, Cbx2 $2^{+/-}$mice produced significantly less IFN- $\beta$ in serum than control mice did (Fig. 3B). Furthermore, the mRNA level of Ifnb was lower (Fig. 3C) and viral titers were higher (Fig. 3D) in macrophages, lungs and livers from $\mathrm{Cbx}^{+/-}$mice versus control mice. Additionally, more lung infiltration of inflammatory cells was observed in $\mathrm{Cbx}^{+/-}$mice than in control mice (Fig. 3E). Accordingly, $\mathrm{Cbx}^{+/-}$mice exhibited a shorter survival time when challenged with VSV (Fig. 3F). Therefore, our in vivo data further confirm that $\mathrm{Cbx} 2$ plays an important role in promoting antiviral innate immune responses.

\section{Cbx2 promotes the transcriptional activation of Ifnb}

To elucidate the underlying mechanism of $\mathrm{Cbx} 2$ in promoting the production of IFN- $\beta$, we examined the effect of $\mathrm{Cbx} 2$ on signaling molecules that are known to function within the viral response pathway, including proximal modulators (RIGI, MAVS, TBK1, MyD88, TRAF3 and TRAF6) and key transcriptional factors (p65 and IRF3/IRF3-5D). The pathways were not significantly impacted by Cbx2-knockdown after infection with either VSV or HSV (Fig. 4A). We next examined the effect of $\mathrm{Cbx} 2$ on the activation of Ifnb promoter by known signaling activators. As shown in Figure 4B and 4C, $\mathrm{Cbx} 2$ significantly enhanced the luciferase activity in IFN- $\beta$ Luc-expressing cells but not in IL-6-Luc-expressing cells after transfection with proximal modulators and key transcriptional factors expression vectors. These results suggest that $\mathrm{Cbx} 2$ enhanced Ifnb transcriptional activation. 


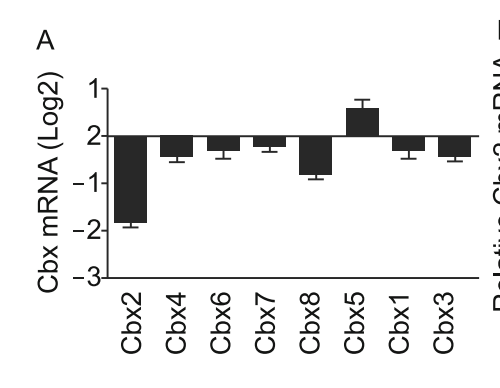

B
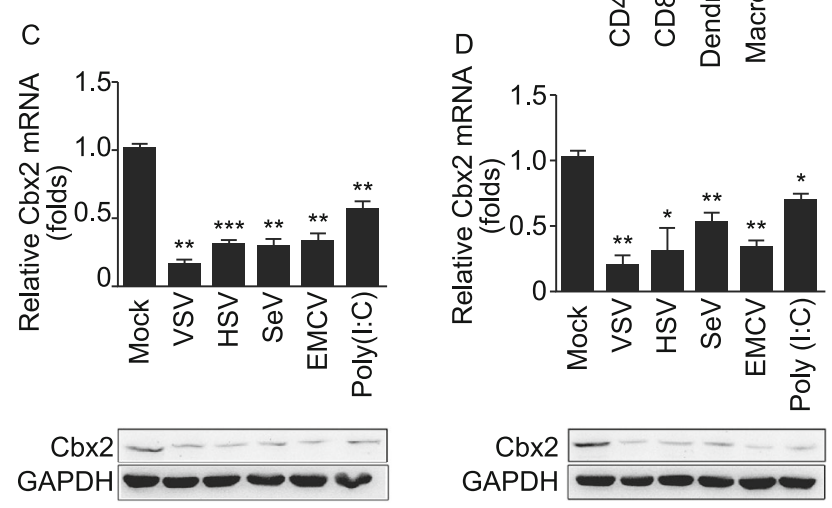

Figure 1. Virus infection down-regulates $\mathrm{Cbx} 2$ expression in macrophages. (A) Q-PCR analysis of CBX family proteins in mouse peritoneal macrophages infected with VSV $(\mathrm{MOI}=10)$ for $8 \mathrm{~h}$. The fold change was calculated relative to the expression in uninfected cells. (B) Q-PCR analysis of Cbx2 in different immune cells. NK, natural killer cells. Expression was normalized to the expression of $\beta$-actin. (C and D) Q-PCR analysis and Western blot of $\mathrm{Cbx} 2$ in mouse peritoneal macrophages (C) or RAW264.7 cells (D) infected with virus $(\mathrm{MOI}=10)$ or incubated with Poly $(\mathrm{I}: \mathrm{C})(100 \mu \mathrm{g} / \mathrm{mL})$ for $8 \mathrm{~h}$. All data are from three independent experiments (mean \pm SEM of technical triplicates) (C and D). Data are representative of three independent experiments with similar results. ${ }^{\star} P<0.05$; ${ }^{*} P<$ $0.01 ;{ }^{* \star *} P<0.001$.

\section{Cbx2 enhances IFN- $\beta$ activation by binding to Jmjd3}

Because Cbx2 is a canonical component of PRC1, which is known to regulate gene expression epigenetically via chromatin modification, we assessed the role of histone modification in the transcriptional activation of IFN- $\beta$. As shown in Figure 5A, infection of macrophages with VSV caused a decrease in the recruitment of H3K27me3 and H3K9me3, and an increase in the recruitment of H3K4me3 to the Ifnb gene promoter. Furthermore, VSV infection caused an increase in the recruitment of $\mathrm{Cbx} 2$ to the Ifnb gene promoter (Fig. 5B). Silencing of $\mathrm{Cbx} 2$ increased the recruitment of H3K27me3 to the Ifnb gene promoter, but had no effect on recruitment of $\mathrm{H} 3 \mathrm{~K} 9 \mathrm{me} 3$ or $\mathrm{H} 3 \mathrm{~K} 4 \mathrm{me} 3$ (Fig. 5C). These results suggest that $\mathrm{Cbx} 2$ suppresses the methylation 3 of H3K27 on the Ifnb gene promoter.

While our findings suggest that $\mathrm{Cbx} 2$ binding to the Ifnb promoter is associated with H3K27 demethylation, Cbx2 is not known to have activity as a demethylase per se.
However, Jmjd3 is known to function as a specifically demethylase for H3K27 (Hong et al., 2007). Therefore, we speculated that Jmjd3 might be involved in Cbx2-mediated suppression of H3K27me3. To evaluate this possibility, we assessed the effect of a small molecule inhibitor of Jmjd3 (HJP). Indeed, Jmjd3-inhibitor decreased the mRNA (Fig. 6A) and protein production of IFN- $\beta$ (Fig. 6B) after VSV infection and also increased the recruitment of $\mathrm{H} 3 \mathrm{~K} 27 \mathrm{me} 3$ to the Ifnb gene promoter (Fig. 6C). Furthermore, overexpression of $\mathrm{Cbx} 2$ enhanced the recruitment of Jmjd3 to the Ifnb gene promoter (Fig. 6D); while conversely, knockdown of Cbx2 suppressed the recruitment of Jmjd3 to the Ifnb gene promoter (Fig. 6E), and this activity could be localized to the proximal $1 \mathrm{~kb}$ to $2 \mathrm{~kb}$ region (Fig. 6F). Moreover, inhibition of Jmjd3 abrogated Cbx2-mediated enhancement of IFN- $\beta$ production (Fig. 6G). Lastly, we performed immunoprecipitation assays to show that $\mathrm{Cbx} 2$ directly bound to Jmjd3 (Fig. 6H).

Taken together, these data demonstrated that $\mathrm{Cbx} 2$ promotes antiviral innate response by binding to and recruiting Jmjd3 to the Ifnb promoter, leading to demethylation of H3K27me3 and increased transcription of IFN- $\beta$.

\section{DISCUSSION}

In this study, we identified that $\mathrm{Cbx} 2$ enhances virus-induced production of type I interferon, and we defined a molecular mechanism for this regulation in which $\mathrm{Cbx} 2$ binds to and recruits Jmjd3 to the Ifnb promoter, leading to demethylation of H3K27 and promotion of Ifnb transcription. Our findings identify a new epigenetic regulator with an integral role in the antiviral innate immune response, which suggests a noncanonical function of $\mathrm{Cbx} 2$.

CBX family proteins play crucial roles in a variety of biological processes by maintaining repressive chromatin states of specific genes. In tumors, CBX family proteins have been shown to endow PRC1 with distinct functions, such as oncogene or tumor suppressor functions. For example, Cbx7 has been identified to act as tumor suppressor in lung carcinoma by suppressing CCNE1 expression (Forzati et al., 2012); conversely, it has been shown to act as an oncogene in gastric cancer by repressing p16 (INK4a) (Zhang et al., 2010). Cbx2 family proteins have also been shown to inhibit neurite development by directly inhibiting neuron-specific gene expression. On the other hand, CBX family proteins also have been shown to enhance gene transcription in certain cases. For example, $\mathrm{Cbx} 4$ enhances the expression of VEGF and promotes angiogenesis in hepatocellular carcinoma cells ( Li et al., 2014a, b), which is consistent with our findings that $\mathrm{Cbx} 2$ enhances antiviral innate immunity by promoting the transcriptional activation of IFN- $\beta$. Therefore, it is possible that these distinct functions of $\mathrm{Cbx} 2$ are cell-typespecific. In tumor cells, Cbx2 is up-regulated and has been identified as a potential drug target (Chen et al., 2017; Clermont et al., 2016), whereas our results demonstrate that $\mathrm{Cbx} 2$ is down-regulated in macrophages infected with virus. 

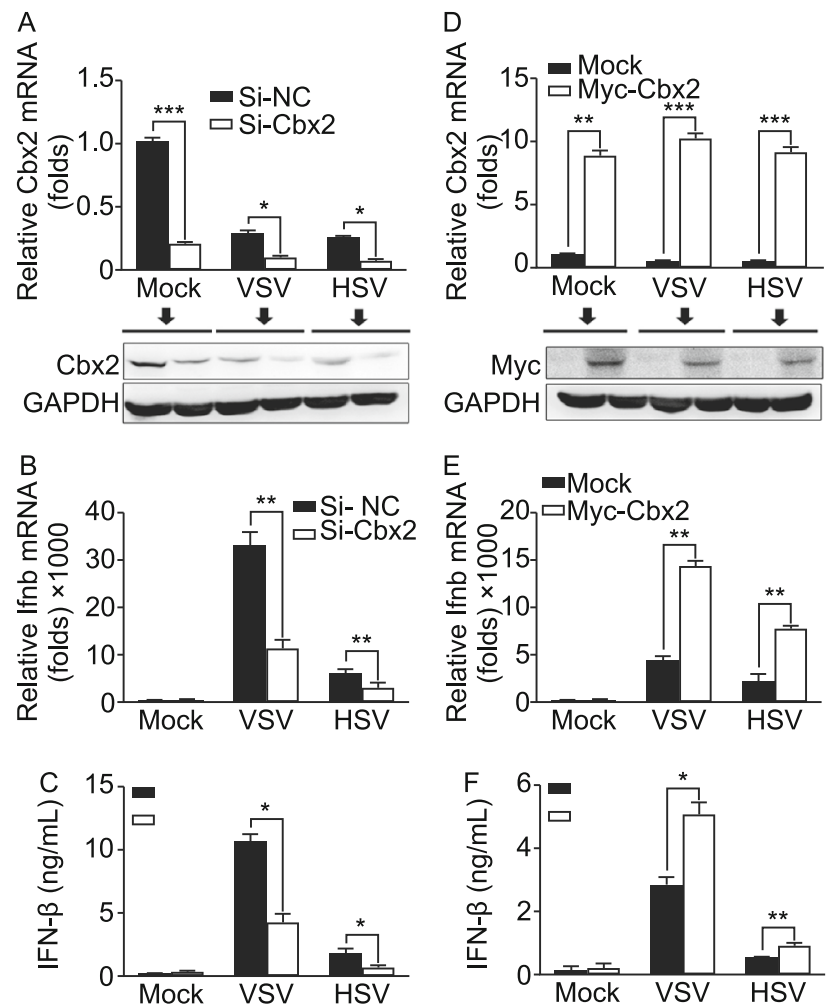

Figure 2. Cbx2 promotes virus-induced type I interferon production in macrophages. Q-PCR (upper panel) and Western blot (lower panel) of $\mathrm{Cbx} 2$ expression in mouse peritoneal macrophages. ( $B$ and $C$ ) Analysis of the level of IFN- $\beta$ mRNA (B) and protein (C) in peritoneal macrophages with Cbx2 knockdown by Q-PCR and ELISA. Cells were transfected with a control siRNA (Si-NC) or a Cbx2-targeting siRNA (Si$\mathrm{Cbx} 2)$ for $48 \mathrm{~h}$, and then infected with virus $(\mathrm{MOI}=10)$ for $8 \mathrm{~h}$ (A-C). (D) Q-PCR (upper panel) and Western blot (lower panel) analysis showing effective overexpression of $\mathrm{Cbx} 2$ in $\mathrm{Cbx} 2$ stably over-expressing (Myc-Cbx2) RAW264.7 cells. (E and F) Q-PCR (E) and ELISA (F) analysis of virus-induced IFN- $\beta$ in Mock or Myc-Cbx2 RAW264.7 cells infected with virus $(\mathrm{MOI}=$ 10) for 8 h. Cbx2 and IFN- $\beta$ mRNA values were normalized to the expression of $\beta$-actin and all data are from three independent experiments (mean \pm SEM of technical triplicates). Data are representative of three independent experiments with similar results. ${ }^{\star} P<0.05 ;{ }^{* \star} P<0.01 ;{ }^{* \star *} P<0.001$.

Therefore, we speculate that the distinct functions of $\mathrm{Cbx} 2$ might be explained both by cell-type-dependent function and expression differences. We also observed that other $\mathrm{Cbx}$ family proteins exhibit decreased expression in virus-infected macrophages, which raises the possibility that other Cbx family proteins might collaborate with $\mathrm{Cbx} 2$ in the antiviral response.

Conventionally, PRC1 proteins bind to $\mathrm{H} 3 \mathrm{~K} 27 \mathrm{me} 3$ and recruit other components of PRC1 to chromatin by proteinprotein interaction to further enhance transcriptional repression of target genes (Gao et al., 2012). However, it has been shown that certain $\mathrm{CBX}$ proteins can associate with moderators other than the PRC1 complex, displaying a PRC1-independent role in transcriptional regulation. For instance, Cbx4 represses the expression of Runx2 and metastasis of colorectal carcinoma, which is dependent on its interaction with HDAC3 (Wang et al., 2016). Furthermore, Cbx8 associates with WDR5, thereby enhancing Notch-related gene expression and promoting breast tumorigenesis (Chung et al., 2016). Intriguingly, our study suggests that Cbx2 enhances IFN- $\beta$ transcription in virus-infected macrophages by inhibiting $\mathrm{H} 3 \mathrm{~K} 27 \mathrm{me} 3$ of Ifnb promoter, which is dependent on the recruitment of $\mathrm{Jmjd} 3$. These findings suggest that the interaction with $\mathrm{Jmjd} 3$ is pivotal for the function of $\mathrm{Cbx} 2$ in antiviral innate immunity. An important issue for the current research is the spatiotemporal dynamics between Cbx2, Jmjd3 and H3K27me3. Furthermore, it would be important to investigate whether other moderator (s) of PRC may be involved in Cbx2-mediated promotion of IFN- $\beta$ transcription.

In $\mathrm{Cbx}^{+/-}$mouse-derived macrophages, increased recruitment of $\mathrm{H} 3 \mathrm{~K} 27 \mathrm{me} 3$ to the Ifnb gene promoter was also observed, suggesting that $\mathrm{Cbx} 2$ reduces $\mathrm{H} 3 \mathrm{~K} 27 \mathrm{me} 3$ at the Ifnb gene promoter. This is in contrast to Polycomb group (PcG) protein-mediated H3K27me3 recruitment to genomic targets (Simon and Kingston, 2013). It has been shown that phosphorylation of $\mathrm{Cbx} 2$ is essential for its transcriptional repression of target genes, and that CK2-mediated phosphorylation inhibits Cbx2's DNA-binding activity and transcriptional repression (Kawaguchi et al., 2017). Given that the expression of CK2 is virus-inducible in macrophages (GEO profile: GDS1271), we speculate that CK2-mediated phosphorylation inhibits recognition and binding to H3K27 in macrophages upon virus infection.

According to reported gene-profiling data, $\mathrm{Cbx} 2$ expression is downregulated in the brain (GEO: GSE44331) and in RAW264.7 macrophages (GEO: GSE81675), which is in agreement with our findings that $\mathrm{Cbx} 2$ expression decreases upon VSV infection. As a positive regulator of IFN- $\beta$ transcription activation, the $\mathrm{Cbx} 2$ expression pattern differs from the expression pattern of other activators that are virus-inducible (Li et al., 2014a). This difference might be explained by a fine-tuning mechanism that serves to prevent superfluous IFN- $\beta$ production. Proper termination of the antiviral effect is necessary to avoid excessive cytotoxicity and tissue injury. Therefore, Cbx2 down-regulation by VSV might constitute a feedback regulation mechanism in the antiviral response.

During virus infection, the host senses components derived from viruses and activates type I IFN response to eliminate viruses. On the other side, viruses have evolved kinds of ways to regulate and evade the host anti-viral immune response, such as sequestering anti-viral signaling transducers and effectors or targeting them for degradation. Furthermore, to establish virus infection-specific expression patterns of host cells for persistent infection or effective 
A

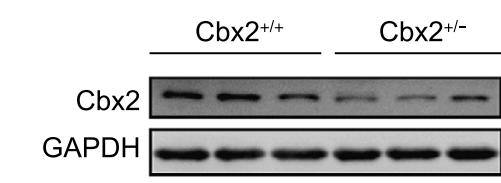

C
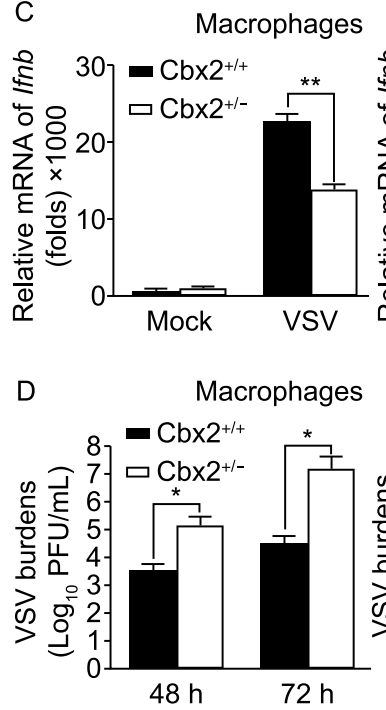

E

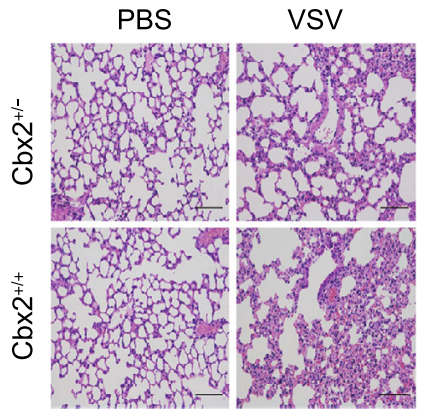

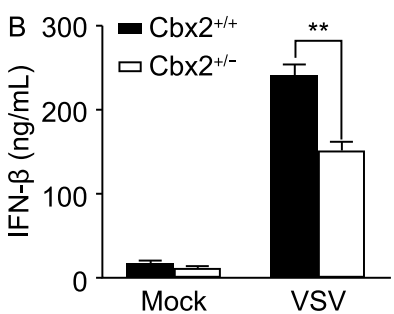

Liver
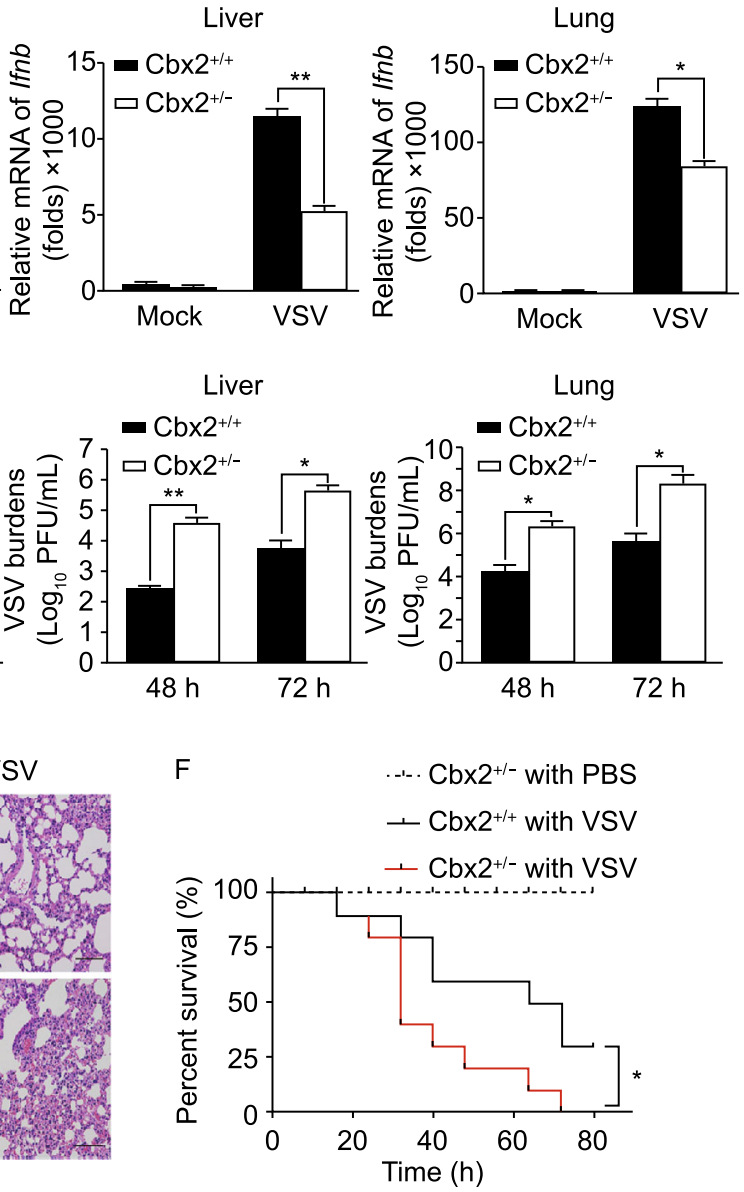

Figure 3. Knockdown of Cbx2 increases susceptibility to VSV challenge. (A) Western blot analysis of Cbx2 expression level in peritoneal macrophages derived from $\mathrm{Cbx} 2^{+/-}$mice as compared to $\mathrm{Cb} \times 2^{+++}$mice. (B) ELISA of IFN- $\beta$ in serum of $\mathrm{Cbx} 2^{+/-}$mice and $\mathrm{Cbx} 2^{+/+}$mice ( $n=6$ per group) after challenge by tail vein injection (IOCV) of VSV $\left(2 \times 10^{6} \mathrm{PFU} / \mathrm{g}\right)$ for $18 \mathrm{~h}$. (C) mRNA level of Ifnb in macrophages, livers and lungs derived from $\mathrm{Cbx} 2^{+/-}$mice or $\mathrm{Cbx}^{+/+}$mice after challenge with VSV $\left(2 \times 10^{6} \mathrm{PFU} / \mathrm{g}\right)$ for $18 \mathrm{~h}$. (D) Plaque assay of VSV burden. Vero cells were infected at a MOI of $0.001 \mathrm{PFU} /$ cell. Viral burdens were quantified by plaque assay in Vero cells after $48 \mathrm{~h}$ and $72 \mathrm{~h}$ and reported as $\log _{10} \mathrm{PFU} / \mathrm{mL}$. (E) Hematoxylin and eosin staining of lung sections from $\mathrm{Cbx} 2^{+/-}$and $\mathrm{Cbx}^{+/+}$mice with or without challenge with VSV $\left(2 \times 10^{6} \mathrm{PFU} / \mathrm{g}\right)$ for $18 \mathrm{~h}$. Scale bar, $100 \mu \mathrm{m}$. (F) Survival of Cbx2 $2^{+/-}$and $\mathrm{Cbx} 2^{+/+}$ mice $\left(n=10\right.$ per group) after challenge with VSV $\left(1 \times 10^{7} \mathrm{PFU} / \mathrm{g}\right)$ for the indicated times. ${ }^{*} P<0.05$ (Log rank test). The data in panels $(B-D)$ is from three independent experiments (mean \pm SEM of technical triplicates). All data are representative of three independent experiments with similar results. ${ }^{*} P<0.05$; ${ }^{\star *} P<0.01$.

replication, virus may inhibit type I IFN response through epigenetic mechanisms (Garcia-Sastre, 2017). In this study, we observed that the expression of $\mathrm{Cbx} 2$, which is a coactivator of IFN- $\beta$, decreased during virus infection, although the underlying mechanism was unclear. Our study implies that virus may inhibit the host type I IFN response through blocking the positive epigenetic mechanism for transcription induction of INF- $\beta$.

In summary, we identified $\mathrm{Cbx} 2$ as a positive regulator for antiviral innate immunity. Our results suggest that $\mathrm{Cbx} 2$ promotes the production of IFN- $\beta$ by associating with Jmjd3 and enhancing its recruitment to the lfnb promoter. Our study 


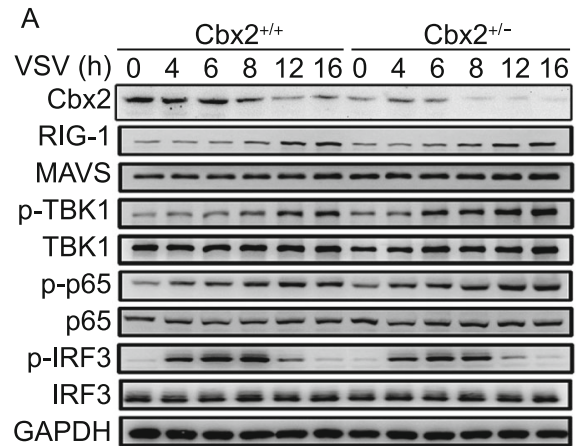

B

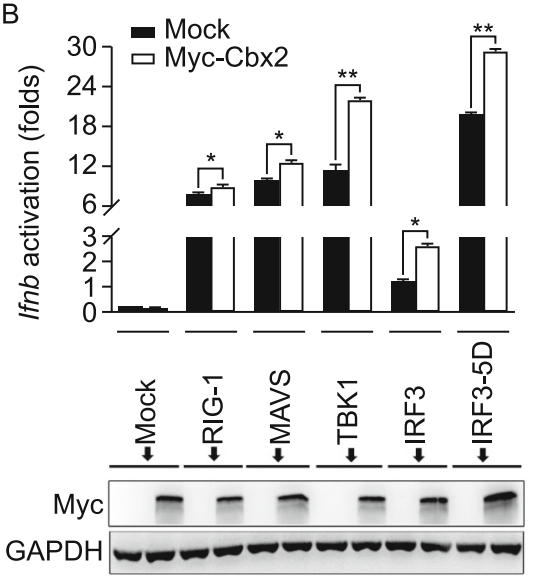

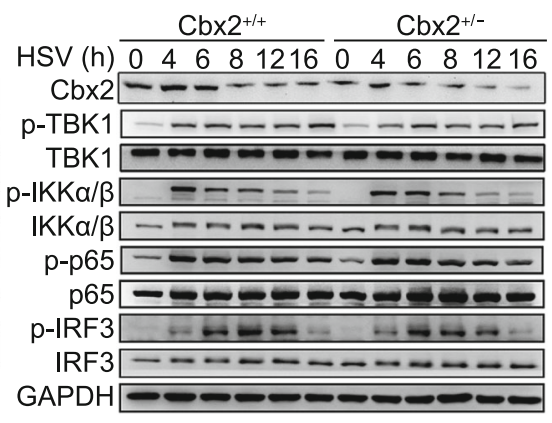
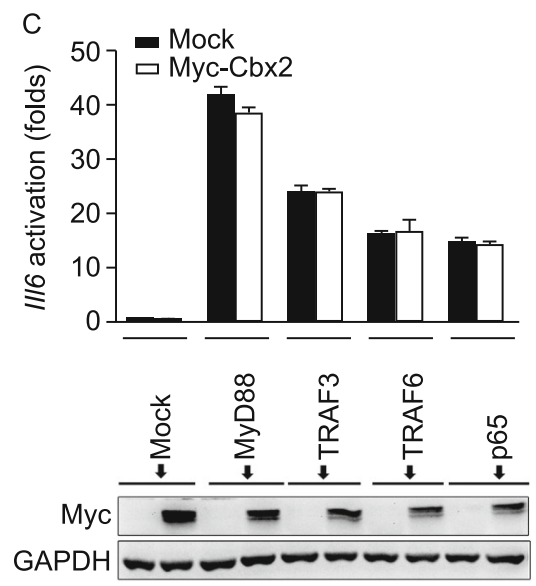

Figure 4. Cbx2 enhances IFN- $\beta$ transcription. (A) Western blot analysis of mouse peritoneal macrophages derived from $\mathrm{Cbx} 2^{+/-}$or $\mathrm{Cbx}^{+/+}$mice after challenge with VSV or HSV $(\mathrm{MOI}=10)$ for the indicated times. $(\mathrm{B}$ and $\mathrm{C})$ Analysis of luciferase activity and mycCbx2 expression in lysates of HEK293T cells stably over-expressing IFN- $\beta$-Luc (B) or IL-6-Luc (C). Results were normalized by Renilla luciferase activity $(n=6)$. Data are representative of three independent experiments with similar results. All data are from three independent experiments (mean \pm SEM of technical triplicates) (B). Data are representative of three independent experiments with similar results. ${ }^{*} P<0.05 ;{ }^{* *} P<0.01$.

provides mechanistic insight into epigenetic modifiers in the regulation of innate immunity.

\section{MATERIALS AND METHODS}

\section{Animals}

$\mathrm{Cbx}^{+/-}$mice were obtained from Jackson Laboratory (Stock No: 003478). The first 4 exons of $\mathrm{Cbx} 2$ were replaced via homologous recombination with a neomycin resistance cassette in reverse orientation. The strain was maintained by heterozygous mating for more than 2 years. Male C57BL/6J mice (six to eight weeks) were from Beijing Vital River Laboratory Animal Technology Co. (Beijing, China). All animals were maintained in specific-pathogen-free conditions. All animal experiments were carried out under the supervision of the Institutional Animal Care and Use Committee (IACUC) of the Institute of Basic Medical Sciences, Chinese Academy of Medical Sciences, Beijing, China.

\section{Plasmid constructs}

A recombinant expression vector encoding N-Myc-tagged Cbx2 was constructed by PCR cloning into EX-Mm01605-M43 eukaryotic expression vector (Fulengene Genecopoeia). The IL-6 and IFN- $\beta$ luciferase reporter plasmids have been described previously ( $\mathrm{Li}$ et al., 2014a). Expression vectors encoding Flag-tagged Cbx2, Myctagged Jmjd3 and Myc-tagged IRF3 were constructed by PCR cloning into pcDNA3.1-Flag or pcDNA3.1-Myc eukaryotic expression vectors. Flag-tagged P65 and IRF3 expression vectors were constructed by PCR cloning into pcDNA3.1 eukaryotic expression vector (Invitrogen, Carlsbad, CA). All constructs were confirmed by DNA sequencing.

Cells and cell transfection

HEK293T and RAW264.7 cell lines were from American Type Culture Collection. Thioglycolate-elicited mouse primary peritoneal macrophages were isolated and cultured, transfected with siRNA pool $(10 \mathrm{nmol} / \mathrm{L})$ through the use of Lipofectamine $\AA$ RNAiMAX Reagent (Thermo Fisher Scientific). The siRNAs targeting mouse Cbx2 were as follow: CAAGUGCGGUCUCGGGCUA, GAGUCAAGUUCUCGUGUUU, UGGCAGAGCUCCAUCGUAC, CCGGGUA ACUGUCUUGAAA (Designed by Dharmacon). For stable overexpression of $\mathrm{Cbx} 2$, we transfected cells expressing the IFN- $\beta$-Luc reporter gene (293T-IFN- $\beta$-Luc cells) or RAW264.7 cells with Myc- 
A

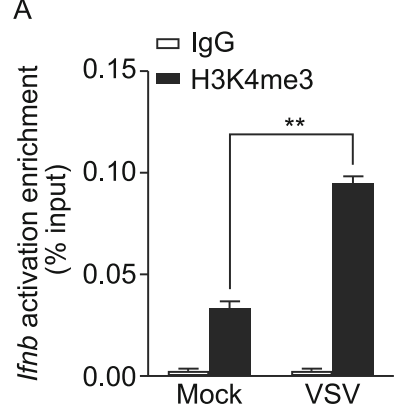

B

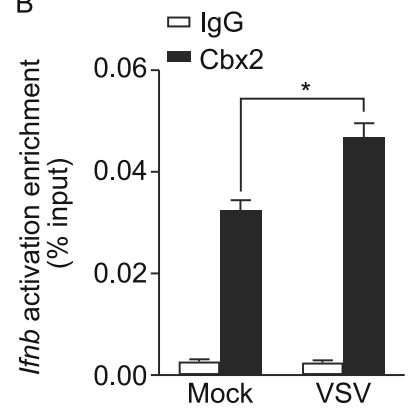

C

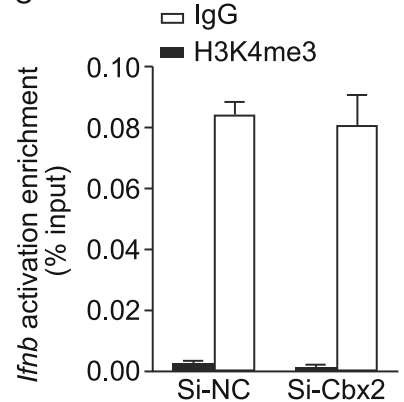

$\square \lg G$
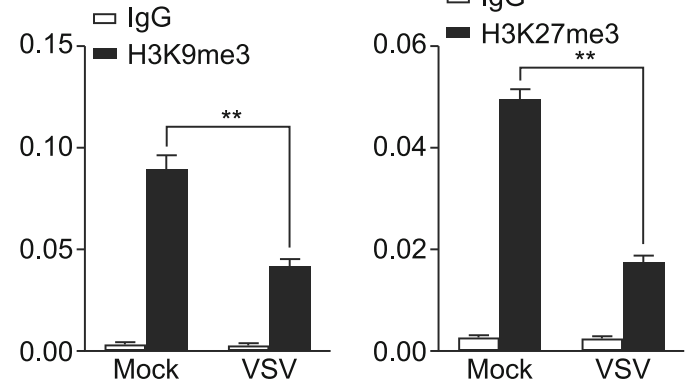

Figure 5. Cbx2 suppresses the methylation3 of H3K27 on the Ifnb promoter. (A) ChIP analysis of methylated histones at the Ifnb promoter in RAW264.7 cells left unstimulated (mock) or infected with VSV (MOI $=10)$ for $6 \mathrm{~h}$. (B) ChIP analysis of Cbx2 at the Ifnb promoter in RAW264.7 cells left unstimulated (mock) or infected with VSV (MOI $=10$ ) for $6 \mathrm{~h}$. (C) ChIP analysis of methylated histones at the Ifnb promoter in mouse peritoneal macrophages transfected with Si-NC or Si-Cbx2 for $48 \mathrm{~h}$, and then infected with VSV $(\mathrm{MOI}=10)$ for $6 \mathrm{~h}$. All data are from three independent experiments (mean $\pm \mathrm{SEM}$ ) of technical triplicates. Data are representative of three independent experiments with similar results. ${ }^{*} P<0.05$; ${ }^{* *} P<0.01$.

Cbx2 and selected the cells in G418 Sulfate ( $800 \mu \mathrm{g} / \mathrm{mL}$; Gibco). For transient transfection of plasmids in HEK293T cells, Lipofectamine ${ }^{\mathrm{TM}}$ 3000 Reagent was used according to manufacturer's instructions (Thermo Fisher Scientific). For transient transfection of plasmids in primary peritoneal macrophages, nuclear transfection was performed by using the AmaxaTMP3 Primary Cell 4D-nucleofectorTM $X$ Kit (Lonza) according to the manufacturer's instructions. All cells were cultured in endotoxin-free DMEM (Gibco), supplemented with 10\% FBS (Gibco), $5 \mathrm{mg} / \mathrm{mL}$ penicillin (Gibco) and $10 \mathrm{mg} / \mathrm{mL}$ streptomycin (Gibco).

\section{Antibodies and reagents}

Antibodies against Myc-tag (2272, 2276, 2040), Flag-tag (14793), phosphorylated IRF3 (Ser396) (\#4947), phosphorylated NF-KB p65
(Ser536) (\#3033), and Normal Rabbit IgG (\#2729) were from Cell Signaling Technology. Antibodies against Cbx2 (LS-C81651, LSC289259) were from LifeSpan BioSciences. Antibodies against Histone H3K27me3 (39155), Histone H3K9me3 (61013), and Histone H3K4me3 (61379) were from Active Motif. Antibodies against $\beta$-actin (M177-3) and GAPDH (M171-3) were from MBL. Protein G-agarose (20397) used for immunoprecipitation was from Pierce; ChIP Grade Protein G Magnetic Beads (\#9006), and Cell Lysis Buffer (\#9803) were from Cell Signaling Technology. Poly (I:C) was from Invitrogen. HSV was a gift from Prof. Q. Li (Chinese Academy of Sciences, Beijing, China), VSV was a gift from Prof. W. Pan (Second Military Medical University, Shanghai, China), and Sendai virus (SeV) was a gift from Prof. B. Sun (Chinese Academy of Sciences, Shanghai, China). HJP (Jmjd3 inhibitor) was a gift from X. Bin 


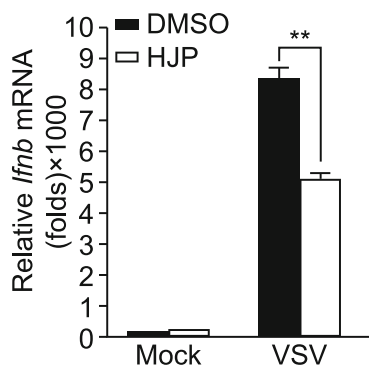

B

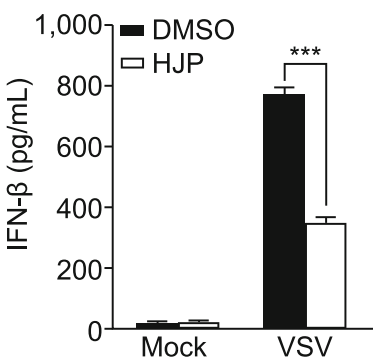

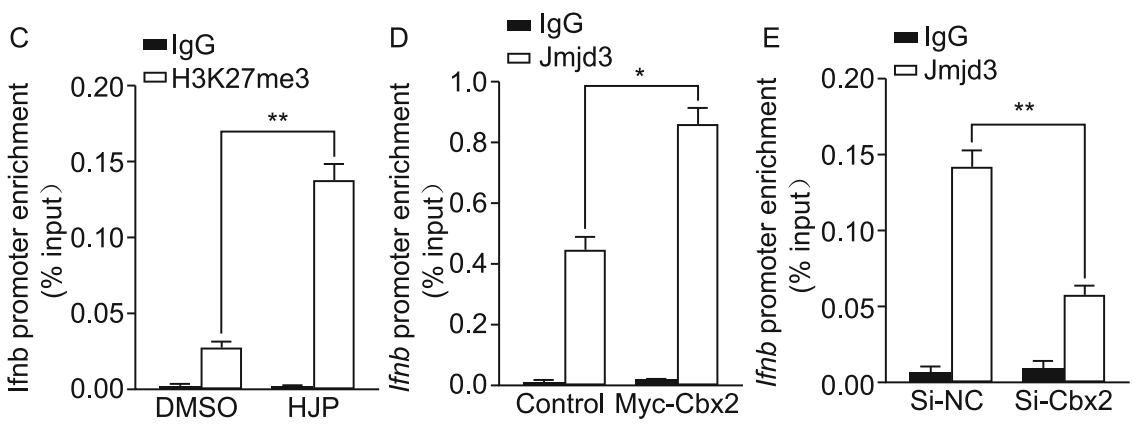

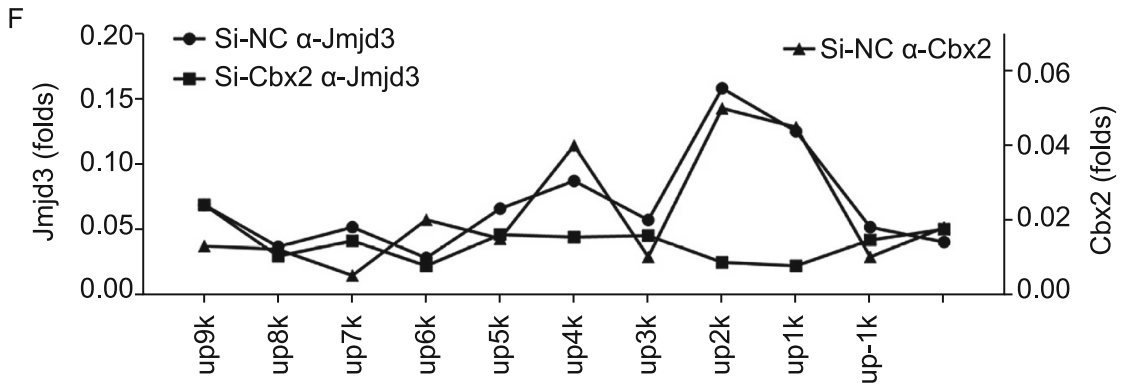

G

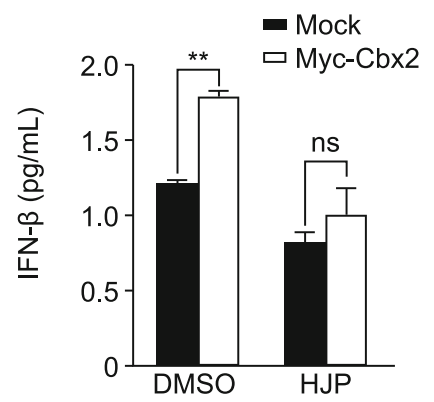

$\mathrm{H}$

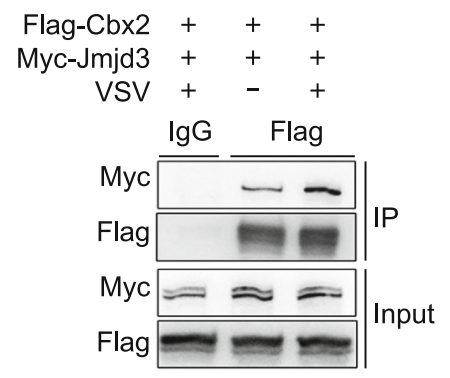

Figure 6. Cbx2 enhances the activation of IFN- $\beta$ activation by binding to Jmjd3. (A) Q-PCR analysis of Ifnb expression, and (B) ELISA analysis of IFN- $\beta$ in supernatants from mouse peritoneal macrophages treated with Jmjd3 inhibitor (HJP) for 6 h, and then infected with or without VSV $(\mathrm{MOI}=10)$ for $6 \mathrm{~h}$. (C) ChIP analysis of H3K27me3 at the Ifnb promoter in mouse peritoneal macrophages treated with HJP for $6 \mathrm{~h}$, and then infected with VSV (MOI = 10) for $8 \mathrm{~h}$. (D and E) ChIP analysis of Jmjd3 at the Ifnb promoter in Myc-Cbx2 RAW264.7 cells (D) or mouse peritoneal macrophages transfected with Si-NC or Si-Cbx2 (E). Cells were infected with VSV $(\mathrm{MOI}=10)$ for $6 \mathrm{~h}$. (F) ChIP analysis of Jmjd3 and Cbx2 at different regions of the Ifnb promoter in control or Cbx2knockdown macrophages. (G) ELISA analysis of IFN- $\beta$ in supernatants mock or Myc-Cbx2 RAW264.7 cells treated with or without with HJP for $6 \mathrm{~h}$. $(\mathrm{H})$ Western blot analysis of the interaction of Cbx2 and Jmjd3 in HEK293T cells transfected with Cbx2 and Jmjd3 expression plasmid. The proteins were immunoprecipitated with IgG or an anti-Flag antibody prior to immunoblotting. The data in panels $(A-G)$ are from three independent experiments (mean \pm SEM of technical triplicates). All data are representative of three independent experiments with similar results. ${ }^{*} P<0.05 ;{ }^{* \star} P<0.01 ;{ }^{* \star *} P<0.001$. 
(Institute of Materia Medica, Chinese Academy of Sciences, Shanghai, China).

\section{Virus infection}

For in vitro virus infection experiments, virus was added along with fresh medium to pre-treated cells for the indicated times at $\mathrm{MOI}$ of 10. For in vivo virus infection experiments, virus was injected via the tail vein.

\section{Quantitative RT-PCR}

Total RNA was extracted with Trizol reagent (Ambion). ReverTra Ace $\AA$ qPCR RT Master Mix with gDNA Remover (Toyobo) was used to synthesize cDNA. KOD SYBR® qPCR Mix (Toyobo) was used for qRT-PCR. Sample data were normalized to GAPDH expression. Primers for qPCR are listed in Table S1.

\section{ELISA}

IFN- $\beta$ and IL- 6 levels in supernatants or sera were measured with mouse IFN- $\beta$ ELISA kits (PBL Biomedical Laboratories).

\section{Immunoblot analysis}

Cells and organs were lysed with RIPA Lysis Buffer (Merck Millipore), and extracted protein was homogenized by the BCA protein assay kit (Pierce). Immunoblot were performed with the indicated antibodies.

\section{VSV plaque assay}

VSV titers of supernatants and tissue homogenate samples were determined by plaque assays on Vero cells. $100 \mu \mathrm{L}$ samples (10-fold dilution) were inoculated onto confluent Vero cell monolayers for $1 \mathrm{~h}$ at $37{ }^{\circ} \mathrm{C}, 5 \% \mathrm{CO}_{2}$, and washed by DMEM. And Vero cells were overlayed with a final concentration of $1 \%$ agar containing MEM, $1 \%$ FBS, $200 \mu \mathrm{g} / \mathrm{mL}$ diethylaminoethyl-dextran, and $0.008 \%$ for $48 \mathrm{~h}$ and 72 h. $0.008 \%$ neutral red was added to the cell monolayers, which were then incubated overnight, and plaques were counted. Viral burdens are reported as the $\log _{10}$ plaque forming units per $\mathrm{mL}$ (PFU/ $\mathrm{mL})$.

\section{Luciferase reporter assay}

293T-IL-6-Luc cells or 293T-IFN- $\beta$-Luc cells were seeded in 96-well plates and then transfected with Cbx2 or empty plasmid and other signaling pathway-related molecular-expressing vectors as indicated, together with TK-Renilla-luciferase as a control. After $36 \mathrm{~h}$ of incubation, whole-cell lysates were collected for measurements of luciferase activity with the Dual-luciferase Reporter Assay System (Promega) according to the manufacturer's instructions. The method has been described previously (Jiang et al., 2018).

Chromatin immunoprecipitation (ChIP)

For ChIP assays, we used ChIP-IT® Express Chromatin Immunoprecipitation Kits (Active Motif) according to the manufacturer's instructions. Primers used for ChIP quantification are listed in Table S2.
Lung histology

Lung tissues from $\mathrm{Cbx}^{+/+}$mice and $\mathrm{Cbx} 2^{+/-}$mice were dissected and processed as described previously (Wang et al., 2017).

Statistical analysis

Statistical significance between two groups was determined by the two tailed Student's $t$-test. Differences were considered to be significant when $P<0.05$. ( ${ }^{\star} P<0.05$; ${ }^{* \star} P<0.01$; $\left.{ }^{* *} P<0.001\right)$. For mouse survival studies, Kaplan-Meier survival curves were generated and analyzed for statistical significance with GraphPad Prism 5.0.

\section{ACKNOWLEDGMENTS}

We thank Ms. Qiaolian Wen and Mrs. Qijiang Cheng for technical assistance. This work was supported by grants from the National Natural Science Foundation of China (Grant Nos. 31770944 and 81788101); the National 135 Major Project of China (2017ZX10102032-002); and CAMS Innovation Fund for Medical Science (2016-I2M-1-003).

\section{ABBREVIATIONS}

Cbx, chromobox 2; H3K27me, histone 3 lysine 27 methylation; IFN$\beta$, interferon beta; IRF3, interferon regulatory factor 3 ; Jmjd3, Jumonji domain-containing protein D3; PcG, Polycomb group; PRC, Polycomb repressive complex.

\section{COMPLIANCE WITH ETHICS GUIDELINES}

Donghao Sun, Xuetao Cao and Chunmei Wang declare that they have no conflict of interest. All institutional and national guidelines for the care and use of laboratory animals were followed. This article does not contain any studies with human subjects performed by any of the authors.

\section{AUTHOR CONTRIBUTIONS}

C. Wang and X. Cao designed and supervised the research; D. Sun performed the experiments, analyzed data and wrote the paper with C. Wang.

\section{OPEN ACCESS}

This article is distributed under the terms of the Creative Commons Attribution 4.0 International License (http://creativecommons.org/ licenses/by/4.0/), which permits unrestricted use, distribution, and reproduction in any medium, provided you give appropriate credit to the original author(s) and the source, provide a link to the Creative Commons license, and indicate if changes were made.

\section{REFERENCES}

Aranda S, Mas G, Di Croce L (2015) Regulation of gene transcription by Polycomb proteins. Sci Adv 1:e1500737 
Bernstein BE, Mikkelsen TS, Xie X, Kamal M, Huebert DJ, Cuff J, Fry B, Meissner A, Wernig M, Plath K et al (2006) A bivalent chromatin structure marks key developmental genes in embryonic stem cells. Cell 125:315-326

Cao X (2016) Self-regulation and cross-regulation of pattern-recognition receptor signalling in health and disease. Nat Rev Immunol 16:35-50

Chen WY, Zhang XY, Liu T, Liu Y, Zhao YS, Pang D (2017) Chromobox homolog 2 protein: a novel biomarker for predicting prognosis and Taxol sensitivity in patients with breast cancer. Oncol Lett 13:1149-1156

Chung CY, Sun Z, Mullokandov G, Bosch A, Qadeer ZA, Cihan E, Rapp Z, Parsons R, Aguirre-Ghiso JA, Farias EF et al (2016) Cbx8 acts non-canonically with Wdr5 to promote mammary tumorigenesis. Cell Rep 16:472-486

Clermont PL, Crea F, Chiang YT, Lin D, Zhang A, Wang JZ, Parolia $A$, Wu $R$, Xue $H$, Wang $Y$ et al (2016) Identification of the epigenetic reader $\mathrm{CBX} 2$ as a potential drug target in advanced prostate cancer. Clin Epigenet 8:16

Di Croce L, Helin K (2013) Transcriptional regulation by Polycomb group proteins. Nat Struct Mol Biol 20:1147-1155

Forzati F, Federico A, Pallante P, Abbate A, Esposito F, Malapelle U, Sepe R, Palma G, Troncone G, Scarfo M et al (2012) CBX7 is a tumor suppressor in mice and humans. J Clin Investig 122:612623

Gao Z, Zhang J, Bonasio R, Strino F, Sawai A, Parisi F, Kluger Y, Reinberg D (2012) PCGF homologs, CBX proteins, and RYBP define functionally distinct PRC1 family complexes. Mol Cell 45:344-356

Garcia-Sastre A (2017) Ten strategies of interferon evasion by viruses. Cell Host Microbe 22:176-184

Hong S, Cho YW, Yu LR, Yu H, Veenstra TD, Ge K (2007) Identification of JmjC domain-containing UTX and JMJD3 as histone H3 lysine 27 demethylases. Proc Natl Acad Sci USA 104:18439-18444

Iwasaki A, Pillai PS (2014) Innate immunity to influenza virus infection. Nat Rev Immunol 14:315-328

Jiang M, Zhang S, Yang Z, Lin H, Zhu J, Liu L, Wang W, Liu S, Liu W, Ma $Y$ et al (2018) Self-recognition of an inducible host IncRNA by RIG-I feedback restricts innate immune response. Cell 173(906919):e913

Katoh-Fukui Y, Tsuchiya R, Shiroishi T, Nakahara Y, Hashimoto N, Noguchi K, Higashinakagawa T (1998) Male-to-female sex reversal in M33 mutant mice. Nature 393:688-692

Kawaguchi T, Machida S, Kurumizaka H, Tagami H, Nakayama J (2017) Phosphorylation of CBX2 controls its nucleosome-binding specificity. J Biochem 162:343-355

Kawai T, Akira S (2010) The role of pattern-recognition receptors in innate immunity: update on Toll-like receptors. Nat Immunol 11:373-384
Li J, Li Y, Cao Y, Yuan M, Gao Z, Guo X, Zhu F, Wang Y, Xu J (2014a) Polycomb chromobox (Cbx) 7 modulates activationinduced CD4+ T cell apoptosis. Arch Biochem Biophys 564:184188

Li J, Xu Y, Long XD, Wang W, Jiao HK, Mei Z, Yin QQ, Ma LN, Zhou AW, Wang LS et al (2014b) Cbx4 governs HIF-1alpha to potentiate angiogenesis of hepatocellular carcinoma by its SUMO E3 ligase activity. Cancer Cell 25:118-131

Li X, Zhang Q, Ding Y, Liu Y, Zhao D, Zhao K, Shen Q, Liu X, Zhu X, Li N et al (2016) Methyltransferase Dnmt3a upregulates HDAC9 to deacetylate the kinase TBK1 for activation of antiviral innate immunity. Nat Immunol 17:806-815

Marcos-Villar L, Diaz-Colunga J, Sandoval J, Zamarreno N, Landeras-Bueno S, Esteller M, Falcon A, Nieto A (2018) Epigenetic control of influenza virus: role of $\mathrm{H} 3 \mathrm{~K} 79$ methylation in interferon-induced antiviral response. Sci Rep 8:1230

McNab F, Mayer-Barber K, Sher A, Wack A, O'Garra A (2015) Type I interferons in infectious disease. Nat Rev Immunol 15:87-103

Santanach A, Blanco E, Jiang H, Molloy KR, Sanso M, LaCava J, Morey L, Di Croce $L$ (2017) The Polycomb group protein CBX6 is an essential regulator of embryonic stem cell identity. Nat Commun 8:1235

Simon JA, Kingston RE (2013) Occupying chromatin: Polycomb mechanisms for getting to genomic targets, stopping transcriptional traffic, and staying put. Mol Cell 49:808-824

Sparmann A, van Lohuizen M (2006) Polycomb silencers control cell fate, development and cancer. Nat Rev Cancer 6:846-856

Wang C, Wang Q, Xu X, Xie B, Zhao Y, Li N, Cao X (2017) The methyltransferase NSD3 promotes antiviral innate immunity via direct lysine methylation of IRF3. J Exp Med 214:3597-3610

Wang X, Li L, Wu Y, Zhang R, Zhang M, Liao D, Wang G, Qin G, Xu $\mathrm{RH}$, Kang $\mathrm{T}$ (2016) CBX4 suppresses metastasis via recruitment of HDAC3 to the Runx2 promoter in colorectal carcinoma. Cancer Res 76:7277-7289

Wu J, Chen ZJ (2014) Innate immune sensing and signaling of cytosolic nucleic acids. Annu Rev Immunol 32:461-488

Yoshida K, Maekawa T, Zhu Y, Renard-Guillet C, Chatton B, Inoue K, Uchiyama T, Ishibashi K, Yamada T, Ohno N et al (2015) The transcription factor ATF7 mediates lipopolysaccharide-induced epigenetic changes in macrophages involved in innate immunological memory. Nat Immunol 16:1034-1043

Zhang Q, Zhao K, Shen Q, Han Y, Gu Y, Li X, Zhao D, Liu Y, Wang C, Zhang $X$ et al (2015) Tet2 is required to resolve inflammation by recruiting Hdac2 to specifically repress IL-6. Nature 525:389393

Zhang XW, Zhang L, Qin W, Yao XH, Zheng LZ, Liu X, Li J, Guo WJ (2010) Oncogenic role of the chromobox protein CBX7 in gastric cancer. J Exp Clin Cancer Res 29:114 